\title{
Tropenophthalmologie
}

\section{Tropical Ophthalmology}

M. Schulze Schwering ${ }^{1}$, P. Kayange ${ }^{2}$

\author{
${ }^{1}$ Universitätsaugenklinik Tübingen \\ ${ }^{2}$ Head of Ophthalmology Unit, University of Malawi, c/o Department of Surgery, College of Medicine, P/Bag 360, \\ Chichiri, Blantyre 3
}

In diesem Beitrag zur Tropenophthalmologie geht es uns nicht darum, die spezifischen Tropenkrankheiten im Einzelnen und die 1999 gegründete Initiative VISION 2020 in ihrer gesamten Bandbreite darzustellen. Das ist bereits in diversen Fachzeitschriften ausführlich geschehen und wir möchten den geschätzten Leser auf diese Beiträge verweisen [1 -4]. Vielmehr geht es uns darum, aufzuzeigen, welche Erfolge insbesondere die Initiative VISION 2020 bereits gezeigt hat, aber auch ihre Schwächen und weitere Herausforderungen sollen herausgearbeitet werden.

\section{Vorbemerkung}

Um weltweit eine qualitativ hochwertige Augenheilkunde jedem Menschen anbieten zu können, bedarf es der sog. „4 A“:

- Awareness,

- Availability,

- Accessibility,

- Affordability.

Wir präsentieren hier ein besonders gelungenes Beispiel aus dem Bundesstaat Andhra Pradesh, Indien. Dort wurde das „Pyramidal Eye Care Service Delivery Model“ entwickelt und erfolgreich implementiert. Um weitere Erfolge in der Bekämpfung von Blindheit und Sehbehinderung zu erreichen, hat die International Agency for the Prevention of Blindness (IAPB) den globalen Aktionsplan für die Jahre 2014-2019 entwickelt. Dieser Plan gibt nicht die genauen Wege der jeweils nationalen Implementierung vor, wohl aber die zu erreichenden Einzelziele mit dem Hauptziel: Verringerung von vermeidbarer Blindheit und Sehbehinderung um 25\% bis 2019 auf Basis der Daten von 2010.

Darüber hinaus machen wir Sie mit Erkrankungen vertraut, mit denen Sie heutzutage in der täglichen Rou- tine eines Augenkrankenhauses im subsaharischen Afrika (SSA) konfrontiert sind:

- Katarakt,

- Glaukom,

- unkorrigierte Refraktionsfehler,

- diabetische Retinopathie,

- Kinderblindheit,

- Hornhautentzündung,

- HIV-assoziierte Augenerkrankungen: CMV-Retinitis, Bindehautkarzinom.

\section{Einleitung}

\section{WHO-Definition der Sehbehinderung und Blindheit}

Es gibt seit 2004 sechs verschiedene Kategorien von Sehbehinderung und Blindheit (Tab. 1). Dabei werden sowohl der Visus als auch eine Einschränkung des Gesichtsfelds eines Patienten erfasst.

Nach heutigem Kenntnisstand erfasste die im Jahr 1999 verwendete ICD-Klassifikation nicht die Anzahl aller blinden und sehbehinderten Menschen. Diese alte Definition ging von einem bestkorrigierten Visus (Best corrected visual Acuity) aus. Damit erfasste dieses 


\section{Abkürzungen}

\begin{tabular}{|c|c|}
\hline ART & antiretrovirale Therapie \\
\hline $\mathrm{CHB}$ & Childhood Blindness \\
\hline CIN & konjunktivale intraepitheliale Neoplasie \\
\hline CMV & Zytomegalievirus \\
\hline CSC & Cataract surgical Coverage \\
\hline CSR & Cataract surgical Rate \\
\hline ECCE & extrakapsuläre Kataraktextraktion \\
\hline EPI & Expanded Program of Immunization \\
\hline FDP & Frequency Doubling Perimetry Technique \\
\hline GIVS & Global Immunization Vision and Strategy \\
\hline GET & Global Elimination of Trachoma \\
\hline $\mathrm{HCW}$ & Health Care Worker \\
\hline HIV & humanes Immundefizienz-Virus \\
\hline HPV & humanes Papillomavirus \\
\hline IAPB & International Agency for the Prevention of Blindness \\
\hline $\mathrm{IOL}$ & intraokulare Linse \\
\hline ITI & Internationale Trachom-Initiative \\
\hline MLW & Mid Level Worker \\
\hline MSICS & Manual small Incision Cataract Surgery \\
\hline NCD & Non-communicable Diseases \\
\hline \multirow[t]{2}{*}{ NGO } & Nichtregierungsorganisationen \\
\hline & (Non-governmental-Organisations) \\
\hline NNO & Neuritis nervi optici \\
\hline NPDR & nichtproliferative diabetische Retinopathie \\
\hline OSSN & Ocular Surface squamous Neoplasia \\
\hline $\mathrm{PHC}$ & Primary Health Care \\
\hline PDR & proliferative diabetische Retinopathie \\
\hline PEWG & primäres Engwinkelglaukom \\
\hline POWG & primäres Offenwinkelglaukom \\
\hline ROP & Retinopathia praematurorum \\
\hline SAFE & $\begin{array}{l}\text { Surgery, Antibiotics, Face Washing, } \\
\text { environmental Improvement }\end{array}$ \\
\hline SSA & subsaharisches Afrika \\
\hline SSI & Sight Savers International \\
\hline TET & Trabekulektomie \\
\hline U5MR & Under-5-Mortality Rate \\
\hline VAD & Vitamin A Deficiency \\
\hline
\end{tabular}

System nicht die Menschen, die dringend einer Brillenkorrektur bedurften, sie aber nicht erhalten konnten. Heute verwendet man den Terminus „tatsächliche Sehkraft“" (Presenting Vision) in einer neuen Klassifikation, die seit 2004 benutzt wird. In Zahlen: Statt wie zuvor 124 Millionen Menschen werden nun 202 Millionen mit einem eingeschränkten Sehvermögen aufgrund unkorrigierter Refraktionsfehler erfasst; das bedeutet 38\% mehr Menschen, die einer Brille bzw. visuellen Rehabilitation bedürfen. 1,18 Millionen von ihnen gelten dem Gesetz nach als blind und 118 Millionen als sehbehindert (Tab. 2).

\section{Von Vision 2020 im Jahr 1999 zum Globalen Aktionsplan im Jahr 2014}

Die Initiative Vision 2020 wurde von der Weltgesundheitsorganisation (WHO), der International Agency for the Prevention of Blindness (IAPB) und Nichtregierungsorganisationen (Non-governmental-Organisations, NGO) 1999 als Initiative zur Verhütung von Blindheit gegründet. Das Ziel war, vermeidbare Blindheit zu bekämpfen.

Die Hauptlast der Blindheit tragen zu 90\% die Entwicklungsländer. 75\% der Blindheit sind vermeidbar (Avoidable), d.h. entweder verhütbar (Preventable) oder behandelbar (Treatable). Die Prävalenz der Blindheit in armen Ländern (1-2\%) war und ist immer noch zehnfach höher als in Industrienationen $(0,1-0,2 \%)$.

\section{Ziele}

Die Initiative VISION 2020 beruht auf drei Zielen:

\section{- Kontrolle der wesentlichen Augenerkrankungen} wie Katarakt, Trachom, Onchozerkose, Erkrankungen im Kindesalter, Refraktionsfehler, Retinopathia diabetica, Glaukom

- Aufbau einer Infrastruktur zur Augenversorgung, wozu Kliniken, Einrichtung, Instrumente, Operationsausrüstung und Bedarf, z. B. Intraokularlinsen und Verbrauchsgüter, auch Brillen, gehören

- Aus- und Weiterbildung der Ärzte und des Hilfspersonals für Augenprogramme

Als Basis zur Beurteilung dienen Zahlen von 2010 (Tab. 2): 285 Millionen Menschen weltweit leiden an einer Sehbehinderung, 39 Millionen von ihnen sind blind. 60\% von ihnen sind Frauen. Weltweit ist der Anteil an Blindheit und Sehbehinderung bei über 50-Jährigen besonders hoch: Von allen Blinden sind $85 \%$ über 50 Jahre und von allen Sehbehinderten sind $70 \%$ über 50 Jahre alt. Hauptursachen einer reversiblen Sehbehinderung sind Refraktionsfehler (43\%) und der graue Star (33\%). Der häufigste Grund einer reversiblen Erblindung ist der graue Star (51\%). Eine Katarakt ist einfach und preiswert zu behandeln und ist eine der kosteneffektivsten Interventionen in der medizinischen Versorgung überhaupt. Refraktionsfehler können mit einer Brille korrigiert werden. 


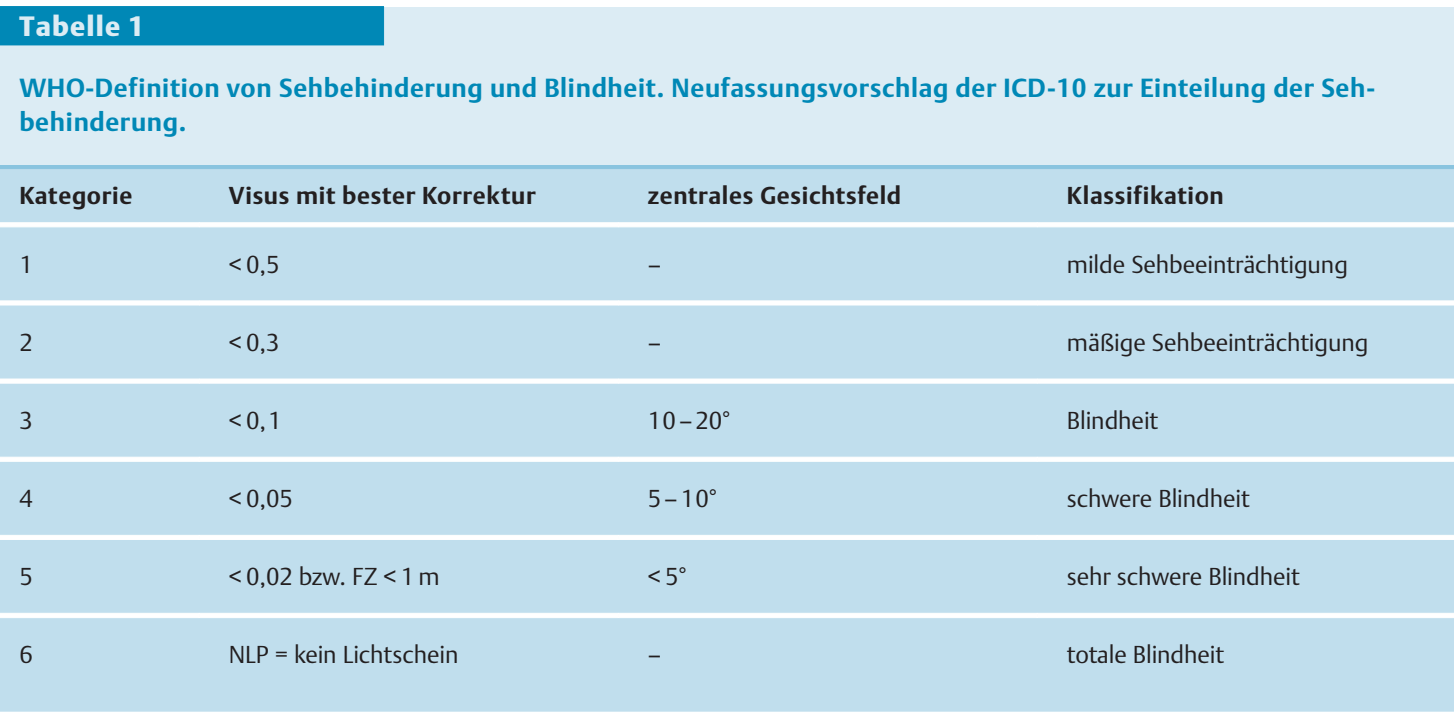

\section{Tabelle 2}

Die Ursachen der Sehbehinderung mit Angabe der Anzahl der betroffenen Personen (geschätzt) im Jahr 2010.

\begin{tabular}{|c|c|c|c|c|}
\hline Erkrankung & $\begin{array}{l}\text { blind } \\
\text { (Mio.)* }\end{array}$ & $\begin{array}{l}\text { eingeschränkte Sehkraft } \\
\text { (Mio.)* }^{*}\end{array}$ & $\begin{array}{l}\text { Sehbehinderung (eingeschränkte } \\
\text { Sehkraft und blind) (Mio.)* }\end{array}$ & $\begin{array}{l}\text { Prozentsatz } \\
\text { (\%) }\end{array}$ \\
\hline unkorrigierter Refraktionsfehler & 1,18 & 118,68 & 119,86 & 42 \\
\hline Katarakt & 20,08 & 74,10 & 94,18 & 33 \\
\hline Glaukom & 3,15 & 2,56 & 5,71 & 2 \\
\hline AMD & 1,97 & 0,89 & 2,85 & 1 \\
\hline Hornhauttrübung & 1,58 & 1,28 & 2,85 & 1 \\
\hline Trachom & 1,18 & 1,67 & 2,85 & 1 \\
\hline diabetische Retinopathie & 0,39 & 2,46 & 2,85 & 1 \\
\hline Kinderblindheit & 1,58 & 1,28 & 2,54 & 1 \\
\hline sonstige & 8,27 & 43,10 & 51,37 & 18 \\
\hline gesamt & 39,37 & 246,02 & 285,39 & \\
\hline
\end{tabular}

Eine weltweite Herausforderung bleibt es, überall gleiche Verhältnisse im Zugang und der Verteilung von Gesundheitsfürsorge zu erreichen. Im englischen Sprachgebrauch nennt man die sogenannten „4 A“ als Basis und Schlüssel für den Erfolg gleicher Verhältnisse im Gesundheitswesen:
- Awareness (Bewusstseinsbildung bzw. Bekanntheitsgrad),

- Availability (Verfügbarkeit oder Betriebsbereitschaft),

- Accessibility (Erreichbarkeit),

- Affordability (Erschwinglichkeit). 

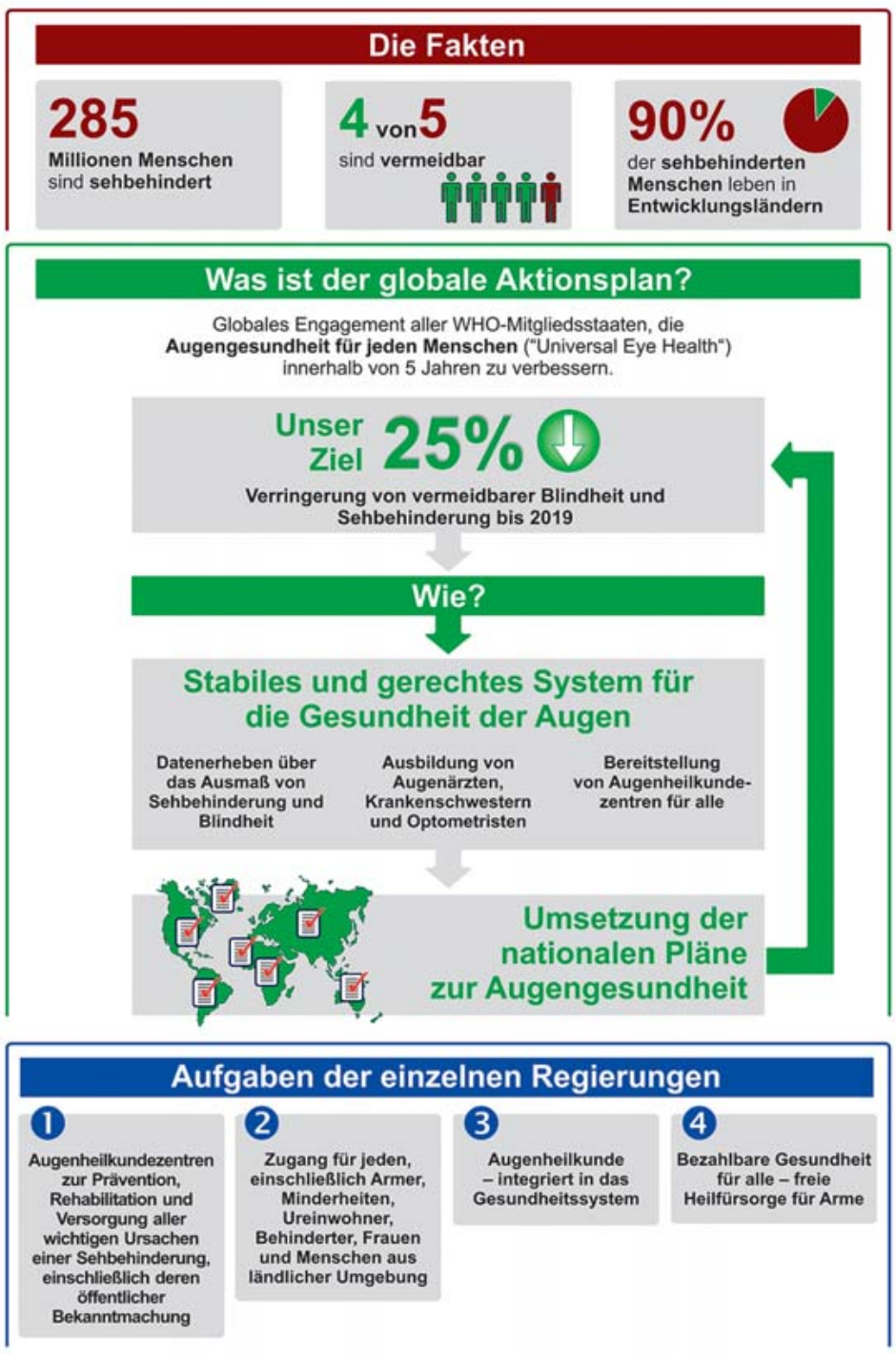

Abb. 1 IAPB - globaler Aktionsplan 2014.

Sie können gleichzeitig sowohl Basis für als auch Barriere zum Erfolg sein. Dies trifft insbesondere für Menschen zu, die in städtischen Slums oder in ländlichen Gegenden wohnen und denen keine besondere Beachtung geschenkt wird. Gesundheitsfürsorge fehlt oft dort, wo arme Menschen wohnen.

Um weitere Erfolge in der Bekämpfung von Blindheit und Sehbehinderung zu erreichen, hat die IAPB den globalen Aktionsplan für die Jahre 2014-2019 festgelegt (Abb. 1). Oleg Chestnow, stellvertretender Direktor für nichtübertragbare Krankheiten (Non-communicable Diseases, NCD) und mentale Gesundheit der WHO betont in seinem Vorwort zum globalen Aktionsplan, dass das Hauptaugenmerk zur Stärkung der Augenheilkunde darin liegen muss, diese stärker in nationale Gesundheitssysteme zu integrieren. Die Mitgliedstaaten verpflichten sich, dass sie die Prävalenz der vermeidbaren Sehbehinderung - basierend auf den Daten von 2010 - um 25\% bis 2019 reduzieren wollen.

\section{Erfolge von VISION 2020 bis zum Jahr 2010}

Nach quasi der Hälfte der Zeit, 2010, nahm man eine erste große Zwischenbewertung vor. Mehr als 100 nationale Pläne zur Eliminierung von Sehbehinderung und Blindheit waren bis dahin aufgestellt worden. So rücken die Probleme Sehbehinderter und Blinder mehr und mehr in den Fokus ihrer Länder und Regierungen. Der globale Trend zu steigernder Prävalenz von Blindheit und Sehbehinderung konnte umgedreht werden und das trotz zahlenmäßig ansteigender und älter werdender Bevölkerung. Die Anzahl der Personen über 50 Jahre stieg um 18\% zwischen 2004 und 2010. Das wahre Ausmaß an Sehbehinderung und Blindheit erfasste man seit 2004 jedoch durch die in Tab. 1 genannte Neudefinition von Sehbehinderung und Blindheit. Diese Erkenntnis führte besonders dazu, dass mehr Optometristen, Optiker und Refraktionisten ausgebildet wurden.

Die Anzahl der Kataraktoperationen stieg weltweit, zum einen durch eine verbesserte Ausbildung, zum anderen durch die preiswerte Produktion intraokularer Linsen (IOLs) für ca. 2 US-Dollar Stückpreis und die Anwendung der manuellen Kleinschnitttechnik (s.u.).

Weltweit gelang es, infektiös bedingte Erblindungen bei Onchozerkose und Trachom deutlich zu reduzieren.

1987 schätzte man, dass 100 Millionen Menschen dem Risiko einer Infektion durch Onchozerkose ausgesetzt waren, 300000 von ihnen waren erblindet und 600000 waren sehbehindert. Die chronische Krankheit der Onchozerkose wird durch Filarien der Art Onchocerca volvulus aus der Gruppe der Fadenwürmer verursacht und kann zur Erblindung führen, der sogenannten Flussblindheit. Der seit 1974 unternommene Versuch, die Onchozerkose durch Vektorkontrolle des Krankheitsüberträgers, Kriebelmücken der Gattung Simulium, zu reduzieren, war gescheitert. Die Firma Merck entwickelte das Medikament Ivermectin zur Abtötung der Mikrofilarien von Onchocerca volvulus und entschied 1987, es solange umsonst zur Verfügung zu stellen, wie es benötigt würde.

Heutzutage bekommen mehr als 80 Millionen Menschen jährlich Ivermectin, was eine immense logisti- 
sche Leistung ist. Wo möglich, erfolgt die Behandlung zu Beginn gleichzeitig mit einer 6-wöchigen Behandlung mit Doxycyclin, das die Sterilisation der weiblichen Würmer bewirkt. Doxycyclin ist gegen die für den Fadenwurm essenziellen Endosymbionten der Gattung Wolbachia gerichtet.

Ein ähnlicher Erfolg gelang in der Bekämpfung des Trachoms. Das Trachom, die sog. ägyptische Körnerkrankheit oder trachomatöse Einschlusskonjunktivitis, ist eine bakterielle Entzündung des Auges mit Chlamydia trachomatis (Serotyp A bis C). Unbehandelt kann sie als chronisch-follikuläre Bindehautentzündung über die Trichiasis (Reiben der Wimpern auf der Hornhaut) zur Erblindung der Hornhaut führen. Seit 1999 sind durch die Internationale Trachom-Initiative (ITI) mehr als 150 Millionen Dosen von Azithromycin verteilt worden. Noch 1990 schätzte man die Zahl der an Trachom erblindeten Menschen auf 5,89 Millionen. 2009 schätzte die WHO 1,18 Millionen Blinde, 1,67 Millionen Sehbehinderte und 40,6 Millionen Infizierte in 57 Ländern, die Endemiegebiete sind. Das Hauptprojekt hierzu ist das WHO Global Elimination of Trachoma by 2020 (GET 2020) mit der SAFE-Strategie (Surgery, Antibiotics, Face Washing, Environmental Improvement).

Es erscheint realistisch, sowohl die Onchozerkose als auch das Trachom in der Zeit zwischen 2020 und 2030 fast ausrotten zu können. Ebenso wurden große Erfolge in der Reduzierung der Kinderblindheit durch Bekämpfung von Masern, Vitamin-A-Mangel und Retinopathia praematurorum (ROP) erreicht (s.u.).

\section{Das System der verschiedenen Gesundheitssektoren}

\section{Primärer Sektor - Grundversorgung}

Grundversorgung bezieht sich auf die Arbeit der Gesundheitsberufe, die eine erste Anlaufstelle für alle Patienten innerhalb eines Gesundheitssystems sind. In Deutschland ist es der Hausarzt, in Entwicklungsländern in der Regel eine Arzthelferin, eine Krankenschwester oder ein Medizinischer Techniker. Dem Ausbildungsstand entsprechend können sie abhängig vom Gesundheitszustand des Patienten ihn an die sekundäre Versorgung weiterleiten oder geringe Krankheiten selbst behandeln.

\section{Sekundärer Sektor (oft synonym: Facharzt- oder Krankenhausversorgung)}

Sekundärversorgung ist die Gesundheitsversorgung durch Fachärzte und andere Gesundheitsberufe, die in der Regel nicht den ersten Kontakt mit den Patienten haben, zum Beispiel Kardiologen, Urologen, Dermatologen usw. Sie umfasst die Akutversorgung, beispielsweise in einer Krankenhausnotaufnahme. Dazu gehören auch die Geburtshilfe, Intensivmedizin und medizinische Bildgebung. Viele sekundären Leistungserbringer müssen nicht unbedingt in Krankenhäusern tätig sein, wie Psychiater, klinische Psychologen, Ergotherapeuten oder Physiotherapeuten.

\section{Tertiärer Sektor}

Tertiärversorgung ist das spezialisiert behandelnde Gesundheitswesen. In der Regel ist es für Patienten ein stationärer Aufenthalt auf Überweisung von einer im primären oder sekundären Sektor tätigen medizinischen Fachkraft. Beispiele für tertiäre Einrichtungen sind Krebszentren, Neurochirurgie, Herzchirurgie, plastische Chirurgie, Behandlung von schweren Verbrennungen, erweiterte Neugeborenendienstleistungen, palliative und andere komplexe medizinische und chirurgische Eingriffe (Abb. 2).

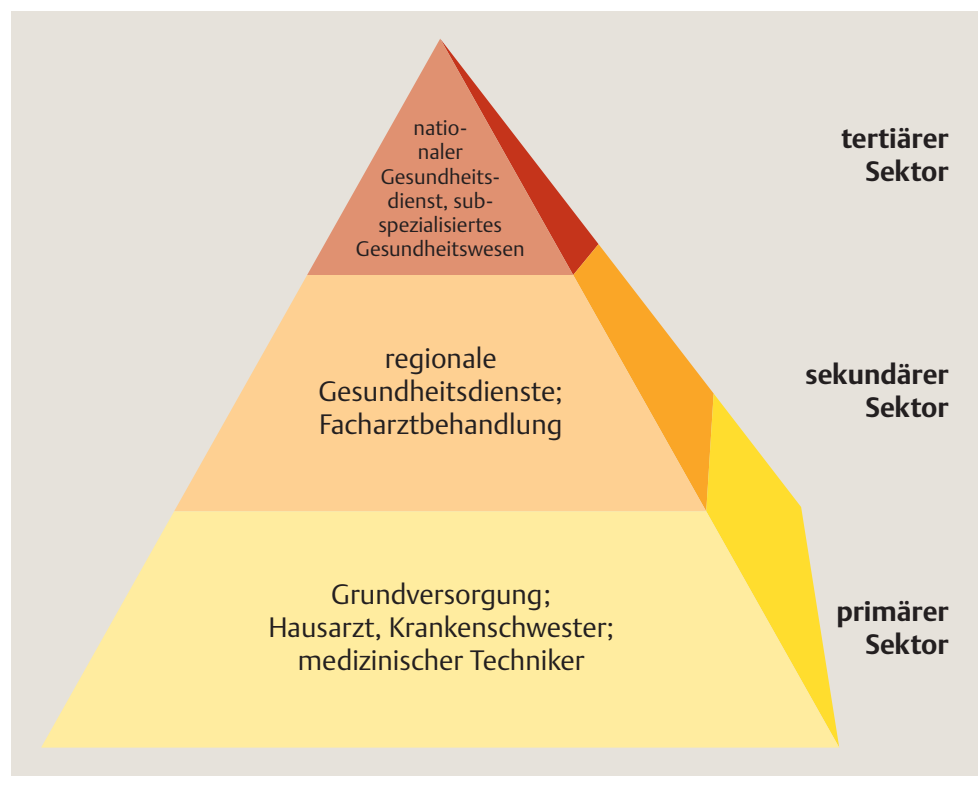

Abb. 2 Pyramide der drei Gesundheitssektoren. 


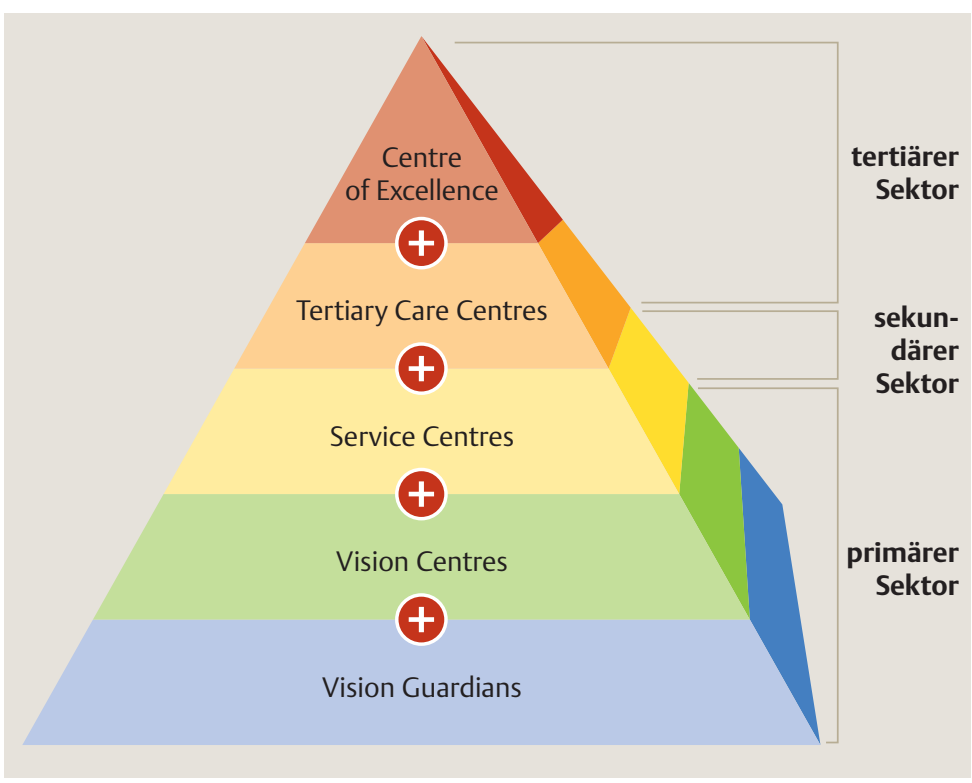

Abb. 3 L. V. Prasad Eye Institute: Pyramidal Eye Care Service Delivery Model (primärer Sektor: blau/grün, sekundärer Sektor: gelb, tertiärer Sektor: orange/rot).

\section{Das L. V. Prasad Eye Institute: Pyramidal Eye Care Service Delivery Model}

Ein Modell, das von verschiedenen Nationen weltweit kopiert werden könnte, ist die vom L.V. Prasad Eye Institute entwickelte Pyramide (Abb. 3, Pyramidal Eye Care Service Delivery Model). Sie besteht aus fünf Schichten, die das primäre (blau/grün), das sekundäre (gelb) und tertiäre (orange/rot) Gesundheitssystem eng miteinander verzahnen.

An der Spitze der Pyramide obliegt es dem Centre of Excellence (Zentrum der umfassendsten medizinischen Versorgung), jede Aktion der untergeordneten Zentren der Augenheilkunde zu koordinieren. Es ist vergleichbar mit dem Niveau einer deutschen Universitätsklinik für Augenheilkunde mit der klassischen Trias von Forschung, Lehre und Krankenversorgung - mit der Besonderheit der Führungsverantwortung der nachgeordneten Zentren.

Direkt darunter arbeitet das Tertiary Care Centre. Es steht für die Versorgung von 5 Millionen Menschen zur Verfügung, jedoch ohne reguläre Forschungsaktivität, dafür aber mit subspezialisierten Ophthalmologen als spezialisierte Katarakt- bzw. Glaukomchirurgen, Kinder- oder Neuroophthalmologen.
Die Basis der Pyramide in diesem Modell wird gebildet von Vision Guardians (Wächter/Hüter des Sehens). Ein Vision Guardian ist verantwortlich für eine Population von ca. 5000 Menschen, oft bestehend aus 1 - 4 Dörfern. Vision Guardians haben einen einfachen Schulabschluss und schauen nach Menschen mit offensichtlichen Erblindungsursachen, um sie dann zum nächsthöheren Zentrum in der Gesundheitspyramide zu senden. Sie entstammen ihrem Versorgungsgebiet, sind ihm emotional verbunden und deshalb sehr engagiert.

Mittlerweile hat das L.V. Prasad Eye Institute unter Leitung von G. N. Rao auch begonnen, Vision Guardians zu Gesundheitserziehern für Diabetes und Bluthochdruck auszubilden. Ein Weg, der auch schon von Kalua in Malawi beschrieben wurde: Dort bildetet man Primary Health Care Worker (PHCW) so aus, dass sie Ursachen einer Erblindung im Kindesalter erkennen können, um so identifizierte Kinder dann zu einem Krankenhaus der Sekundär- oder Tertiärversorgung weiterleiten zu können.

Besonderes Augenmerk in der Ausbildung wird auf das Erkennen des sog. „White Eye“ (Leukokorie) gelegt. Zu den Differenzialdiagnosen gehören u.a.:

- Katarakt,

- korneale Erblindung durch ein kongenitales Glaukom oder ein Trauma,

- Retinoblastom,

- Netzhautablösung,

- primärer hyperplastischer Glaskörper,

- Toxocara-Infektion,

- Morbus Coats.

Vision Center (Sehzentrum) gewährleisten eine ophthalmologische Primärversorgung. Es arbeiten dort sogenannte Techniker (Ophthalmic Technicians) für ca. 50000 Menschen, die ein einjähriges Training in BasisAugenheilkunde und Refraktion absolviert haben. Sie behandeln einfache Bindehautentzündungen und korrigieren leichtere Refraktionsfehler. Für komplexere Pathologien bzw. Operationen des grauen oder grünen Stars überweisen sie an sogenannte Service Center (Service-Zentren) des sekundären Sektors, vergleichbar einer aufgewerteten deutschen operativen Tagesklinik für Augenheilkunde mit integriertem Optiker. Dort werden auch die diabetische Retinopathie und Infektionen wie z. B. Uveitiden behandelt. Zudem werden vergrößernde Sehhilfen angepasst. 


\section{Investitionen in die Augengesundheit}

Das Hauptziel der Initiative Vision 2020 ist es, weltweit eine gleich hohe und umfassende Qualität der Augenheilkunde anzubieten. Diese soll für alle Menschen den gleichen Qualitätsstandard haben und einfach zugänglich sein. Das Pyramidenmodell aus Indien hat gezeigt, dass es am besten in Gebieten mit starker Bevölkerungsdichte funktioniert. Es muss demnach bei geringerer Populationsdichte und entsprechend nationalen Besonderheiten angepasst werden.

Es lohnt sich aber nicht, nur auf die Verbesserung der Augengesundheit alleine zu schauen, sondern es lohnt sich auch finanziell für Regierungen, in die Augengesundheit zu investieren. Dies hat die Fred Hollows Foundation 2013 untersucht [5]. Der Zugewinn an Produktivität, der durch gutes Sehen in der arbeitenden Bevölkerung (15-65 Jahre alt) entstünde, liegt in Entwicklungsländern 2,1-mal über den Investitionskosten. Der Nutzwert über einen Zeitraum von 2011 - 2020 wird global auf wenigstens 843,5 Milliarden US-Dollar geschätzt. Dies übersteigt deutlich die Kosten der Investitionen von 394,2 Milliarden US-Dollar. Der Fred Hollows Report besagt, dass ungefähr 84\% der Weltbevölkerung in Entwicklungsländern wohnen, aber nur ein Drittel des totalen globalen Investments dort benötigt wird (s. Tab. 3). Das bedeutet eine Investition von 2,2 US-Dollar pro Person und Jahr.

\section{Ausbildung von Ophthalmologen, Pflegern und Technikern}

Entwicklungsländer leiden an einem starken Mangel an Ophthalmologen. Tab. 4 erläutert die Ziele von VISION 2020 in Bezug auf ein Mindestmaß an Personal, um die gesteckten Ziele erreichen zu können:

Weniger als $1 \%$ aller weltweit tätigen Ophthalmologen arbeitet in Afrika. Fast alle afrikanischen Länder haben weniger als 50\% der von der WHO empfohlenen Mindestanzahl an Ophthalmologen (Tab. 4) Kasper u. Bajunirwe haben herausgearbeitet, dass das subsaharische Afrika zwar 25\% der gesamten Weltlast an Erkrankungen zu tragen hat, aber nur 3\% des weltweiten Gesundheitspersonals (Health Care Worker, HCW) besitzt [7]. 32 Länder im SSA können noch nicht einmal das von der WHO geforderte Minimum von $23 \mathrm{HCW} /$ 10000 Einwohner stellen.

Einen Mangel an Fachärzten aller Disziplinen gibt es schon seit Jahren in Entwicklungsländern. Kurz nach der Kolonialzeit in der Mitte des 20. Jahrhunderts wurden in Afrika häufig Schulen zur Ausbildung von medizinischem Personal im mittleren Bildungssektor gegründet. Dies geschah aber nur, um in kürzerer Zeit dem eklatanten Mangel an Ärzten entgegenwirken zu können. Das Personal dieses Mittelbaus (z. B. Mid Level Worker, MLW), das weniger theoretisches als praktisches Training erhielt, ist in der Behandlung von Krankheiten deutlich eingeschränkter tätig als ein Facharzt. Es ist gut mit den häufigen Krankheiten vertraut. So ersetzt es aber scheinbar oft den Facharzt in Gebieten, wo es keinen Arzt gibt oder eben dort, wo

\section{Tabelle 3}

Summe der globalen Kosten pro Sektor (2011 - 2020) in Milliarden US-Dollar (modifiziert nach [5]).

\begin{tabular}{|c|c|c|c|}
\hline Gesundheitssektor & $\begin{array}{l}\text { Gesamtkosten } \\
\text { (Milliarden US \$) }\end{array}$ & $\begin{array}{l}\text { Industrienationen } \\
\text { (\% Anteil der Gesamtkosten) }\end{array}$ & $\begin{array}{l}\text { Entwicklungsländer } \\
\text { (\% Anteil der Gesamtkosten) }\end{array}$ \\
\hline Bevölkerungsanteil (2010) & & $16 \%$ & $84 \%$ \\
\hline Investition in den primären Sektor & 308,4 & $251,8(82 \%)$ & $56,6(18 \%)$ \\
\hline Investition in den sekundären Sektor & 62,7 & $4,7(7 \%)$ & $58(93 \%)$ \\
\hline $\begin{array}{l}\text { Investition, um den Rückstand an vermeidbarer Blindheit } \\
\text { und Sehbehinderung zu behandeln }\end{array}$ & 23,1 & $9,5(41 \%)$ & $13,6(59 \%)$ \\
\hline Summe & 394,2 & $266,0(67 \%)$ & $128,2(33 \%)$ \\
\hline
\end{tabular}




\section{Tabelle 4}

VISION 2020 - Personalbedarf der konservativen und operativen Augenheilkunde für das subsaharische Afrika (SSA) (modifiziert nach [6]).

VISION 2020: Personalvorgabe (pro 1 Mio. Bevölkerung)

Augenärzte

4

Kataraktchirurgen

10

OCO - Ophthalmic clinical Officer

„Techniker“

Pfleger/Schwestern in der Augenheilkunde

Optometristen

20

Refraktionisten (mittleres Niveau)

CSR - Cataract surgical Rate

2000

(Kataraktoperationen/Millionen/Jahr)

Kataraktoperationen/Chirurg

500

auch kein Arzt arbeiten möchte. MLW sind eine unverzichtbare Stütze des afrikanischen Gesundheitswesens. Auf ihrem Rücken wird die Hauptlast der Gesundheitsversorgung Afrikas erbracht. In dicht besiedelten Gebieten sind sie eine wesentliche Hilfe der Fachärzte. Sie sind offiziell von ihren Landesregierungen anerkannt und können sich auch in fast allen gängigen Fachrichtungen der Medizin spezialisieren.

Eine Zeit lang wurden afrikanische Ärzte im Ausland ausgebildet und nicht selten blieben sie nach ihrer Ausbildung im Gastland. Deshalb wurde z. B. an der Universität von Nairobi ein regionales Trainingszentrum errichtet, an dem Afrikaner verschiedenster Länder ausgebildet wurden. In Bezug auf die Augenheilkunde sind am Kenyatta National Hospital mittlerweile fast 200 Augenärzte ausgebildet worden, die überall in Afrika tätig sind. Inzwischen entstehen auch in anderen Ländern des SSAs eigene Trainingszentren der Tertiärversorgung für Augenheilkunde, wie z. B. in Malawi. Dadurch wird nun schneller eine höhere Konzentration von auch subspezialisierten Fachärzten für Augenheilkunde (Glaukomspezialist, Kinderophthalmologe, Orbitachirurg, Neuroophthalmologie) erreicht. Dennoch wird es noch viele Jahre dauern, in denen die ärztliche Arbeit vom Personal des mittleren Sektors geleistet werden muss, insbesondere bei stetig steigenden Bevölkerungszahlen.
Kombinierte Ausbildungsprogramme in der Augenheilkunde bestehen aus einem Hauptanteil der Ausbildung (11 von 12 Monaten) in Afrika, gepaart mit 4-wöchigen Aufenthalten (1 von 12 Monaten) an der Universitätspartnerklinik in Deutschland. Ein Beispiel hierfür ist die Universitätspartnerschaft der Augenklinik München, LMU mit der Universität von Nairobi, Kenia, die seit 1978 fast 200 junge afrikanische Augenärzte hervorgebracht hat. Ein weiteres Beispiel ist die Partnerschaft der Universitätsaugenklinik Tübingen mit dem College of Medicine, Blantyre, Malawi, die seit 2005 besteht. Sie hat bisher 4 malawische Augenärzte ausgebildet, 5 weitere sind in Ausbildung. Afrikanische Kollegen, die auf diese Art ausgebildet werden, zeigen eine hohe Standorttreue. Im Fachjargon heißt das „Low Brain Drain“, was bedeutet, dass die besten Köpfe des Landes nicht ins meist westliche Ausland nach ihrer Facharztausbildung abwandern.

Auf diese Weise gewöhnen sich afrikanische Assistenzärzte an die lokalen Gegebenheiten mit dem häufigen Mangel an Medikamenten und Verbrauchsmaterialien und oft ärgerlichen technischen Defekten ihrer Instrumente. Im Gastland hingegen erwartet sie eine andere Kultur der Medizin. Mitentscheidend für das Verbleiben im Heimatland ist die stärkere berufliche und familiäre Sozialisation während der 4-jährigen Ausbildung. Eine Schwäche dieser Programme bleibt, dass sie zumeist teuer aus dem Ausland über Jahrzehnte finanziert werden. Somit fehlt der Baustein, dass sie von ihren eigenen Gesundheitsministerien oder lokalen Sponsoren getragen werden könnten, um gänzlich autark zu werden.

\section{Der altersbedingte graue Star - die Katarakt}

Der altersbedingte graue Star ist seit Jahrzehnten in Entwicklungsländern die reversible Haupterblindungsursache Nummer Eins. Nach globalen Schätzungen sind daran weltweit 20 Millionen Menschen erblindet und 90\% von ihnen leben in Entwicklungsländern. Die einzig wirksame Therapie ist eine Operation mit Implantation einer Kunstlinse.

\section{Cataract surgical Rate und Cataract surgical Coverage}

Schon 2001 betonte Allen Foster von der London School of Hygiene and Tropical Medicine, dass es das Ziel sein müsse, genügend Katarakte pro Jahr zu ope- 


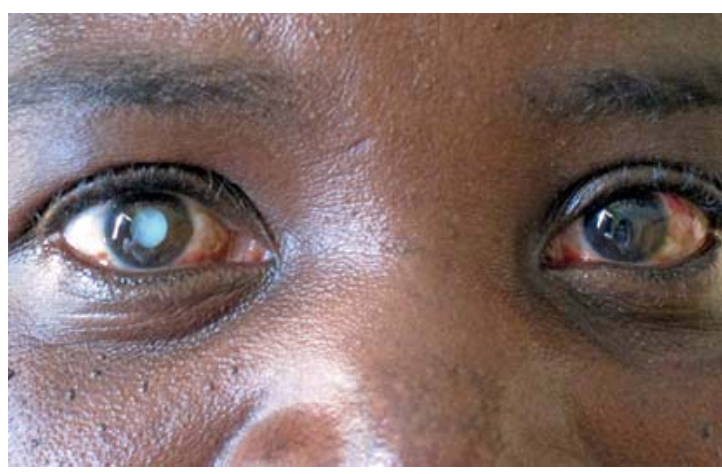

Abb. 4 Afrikanischer Patient mit maturer Katarakt rechts und Pseudophakie links nach Kataraktchirurgie in Kleinschnitttechnik (Manual small Incision Cataract Surgery, MSICS).

rieren, gemessen an der „Cataract surgical Rate“ (CSR), der Anzahl von Operationen pro Jahr pro Millionen Bevölkerung [8]. Da aber der Rückstand (engl. Backlog) der noch zu operierenden Katarakte hoch ist, kann nicht jeder Betroffene eine Operation erhalten, denn das entspräche einer Deckung von 100\% (engl. Coverage). Um nun zu beurteilen, wie viele Bedürftige denn operiert werden, bemüht man den Parameter „Cataract surgical Coverage“ (CSC). Die CSC stellt den Anteil der operierten Patienten geteilt durch die Anzahl der Operierten plus die Anzahl der noch Wartenden dar.

Zehn Jahre später (2011) stellte man fest, dass immer noch mehr als 80\% der Erblindungen bei Menschen über 50 Jahre diagnostiziert werden. Die CSC variiert sehr stark in den Entwicklungsländern. Die WHO empfiehlt eine CSC von mindestens $85 \%$. Schnell durchgeführte Untersuchungen zur Blindheit in Sambia, Nigeria und Malawi ergaben eine entsprechende CSC von 54,46 bzw. $45 \%$.

Es gibt eben vielerlei Hemmnisse in Entwicklungsländern, die zu geringen Operationszahlen führen:

- Angst vor der Chirurgie,

- Kosten,

- langer Anreiseweg zum Hospital,

- weibliches Geschlecht,

- ungebildet oder Analphabet zu sein,

- Leben in ländlicher Gegend,

- noch guter oder bereits postoperativ guter Visus in einem Auge.

Hohe CSC-Raten werden nur aus Ballungsgebieten berichtet, wo auch die CSR hoch ist.

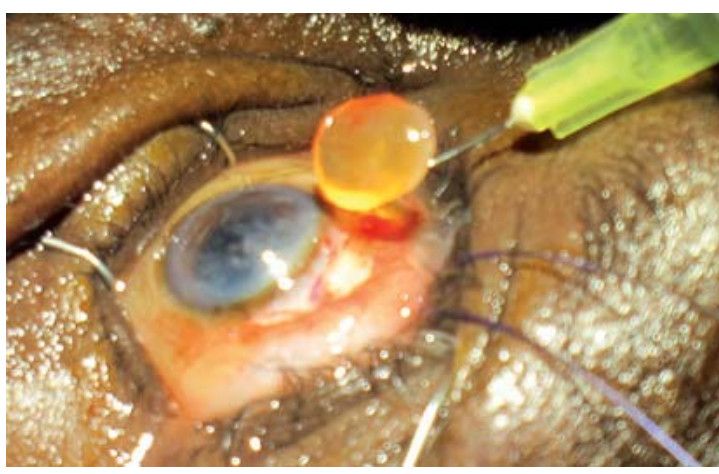

Abb. 5 MSICS - Entfernung des Linsenkerns mit dem „Fishhook“.

\section{Extrakapsuläre Kataraktextraktion, Phakoemulsifikation, manuelle Kleinschnitttechnik}

Derzeit werden weltweit hauptsächlich drei Kataraktoperationstechniken angewendet. Alle drei sind sog. extrakapsuläre Techniken, d.h. die Linsenkapsel bleibt während der Operation erhalten. In diese erhaltene Linsenkapsel wird eine neue künstliche IOL eingepflanzt.

Unter extrakapsulärer Kataraktextraktion (ECCE) versteht man eine Technik, bei der fast ein Drittel der Hornhaut am Limbus eröffnet wird, durch diese Öffnung wird die trübe Linse entfernt und nach Implantation der IOL wird die Hornhaut wieder vernäht.

Nahtlose Techniken sind Phakoemulsifikation (Phako) und manuelle Kleinschnitttechnik (Manual small Incision Cataract Surgery, MSICS):

- Bei der Phakoemulsifikation werden Linsenkern und -rinde mittels Ultraschall durch einen 2-3 mm kleinen, selbstabdichtenden Hornhautschnitt zerkleinert und abgesaugt. Es erfolgt die Implantation einer gefalteten IOL durch diesen kleinen Tunnelschnitt.

- Die MSICS ist die bevorzugte Technik in Entwicklungsländern (Abb. 5 u.6). Es ist ebenfalls eine nahtlose Technik, bei der durch einen korneoskleralen Tunnel die getrübte Linse mittels einer gebogenen Nadel (engl. Fishhook) entfernt wird (Abb. 5). Vorteile sind kurze OP-Dauer, geringe Kosten, schnelle Wiederherstellung des Sehvermögens. Sie zeichnet sich zudem durch eine selbstheilende korneosklerale Wunde nach manueller Entfernung von Kern und Linsenrinde aus. In den Händen eines geübten Chirurgen ist sie innerhalb von Minuten durchzufüh- 


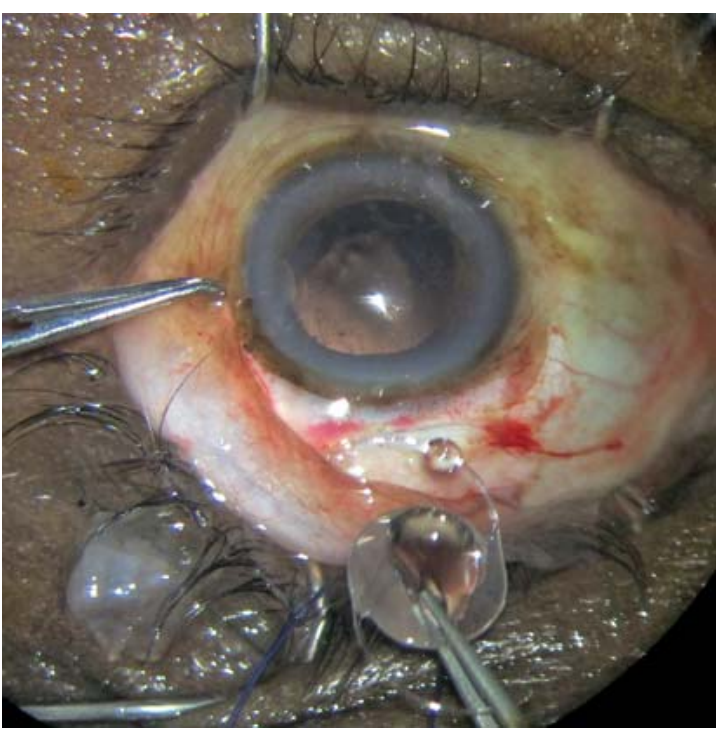

Abb. 6 MSICS - vor Implantation einer Hinterkammerlinse.

ren, bei harten Kernen oft schneller und hornhautschonender als mit der Phakotechnik.

Die visuellen Ergebnisse der beiden Techniken sind vergleichbar.

In einigen Landstrichen Afrikas sind die Ergebnisse nach Kataraktchirurgie für die Patienten unzureichend. Dies liegt häufig an mangelndem Training der Operateure, fehlerhaftem OP-Besteck, mangelhafter Hygiene oder Nichtvorhandensein aller Verbrauchsgüter. Studien aus Sambia und Kenia berichteten von schlechten Ergebnissen bei 31 bzw. 22\% der Patienten.

Die WHO-Richtlinie empfiehlt zur Erreichung der VISION-2020-Ziele, dass zumindest $85 \%$ der operierten Augen eine Sehkraft von mehr als 30\% unkorrigiert erreichen sollen. Zwar erscheinen 30\% Sehkraft sehr wenig, doch $30 \%$ stellen die unterste WHO-Kategorie für „Gutes Sehvermögen“ dar. Um sich diese Prozentzahl besser vorstellen zu können, bedenke man, dass bei uns in Deutschland viele Kataraktpatienten zur Augenuntersuchung mit dem Auto fahren und dann einen Visus von $50 \%$ auf dem gut sehenden und $30 \%$ auf dem schlechter sehenden Auge bei Erstvorstellung präsentieren. Diese Patienten meinen oft, dass man das Auge mit 50\% Sehkraft noch gar nicht operieren müsste. Wie schlecht sie damit tatsächlich sehen, merken sie erst, wenn das 30\% sehende Auge postoperativ wieder 100\% sieht.
Die Erblindung durch die Katarakt wird heute und in Zukunft eine logistische Herausforderung bleiben, speziell bei immer weiter alternder Weltbevölkerung.

Merke: Die CSR bleibt der Indikator für die Verfügbarkeit und Akzeptanz einer operativen Einheit. Jedoch wird nur die gelungene, effektive Kombination von CSR und CSC zum Ziel der Eradikation vermeidbarer Katarakterblindung führen.

\section{Unkorrigierte Refraktionsfehler}

670 Millionen Menschen haben weltweit keine Brillen: 517 Millionen haben einen myopen, 153 Millionen einen hyperopen Refraktionsfehler. Insgesamt 120 Millionen von ihnen gelten als sehbehindert. Die Prävalenz der unkorrigierten Refraktionsfehler ist besonders stark durch sozioökonomische Faktoren bestimmt. Der Bedarf zur Refraktionskorrektur ist unter Kindern am höchsten. Ein Refraktionsdefizit als alleinige Ursache einer Sehbehinderung wird mit 55-83\% aus Indien, China, Nepal und Chile berichtet.

Smith u. Kollegen haben den Produktivitätsverlust der globalen Weltwirtschaft als Folge eines Refraktionsfehlers aus 269 Millionen US-Dollar pro Jahr beziffert [9].

Naidoo u. Jaggernath fordern aufgrund der beschriebenen Probleme eine Verstärkung der personellen Mittel zur Ausbildung von Refraktionisten, den Aufbau entsprechender Infrastruktur zur Refraktion und die Einbeziehung dieser optischen Services in bereits bestehende Augenheilkundeprojekte [10]. All diese Forderungen sind im L.V. Prasad Eye Institute Pyramidal Eye Care Service Delivery Model bereits vom primären Sektor an erfüllt (s.Abb. 3).

Ein weiterer Baustein, um mehr Menschen mit bezahlbaren Gläsern zu versorgen, ist das vom Physiklehrer Martin Aufmuth entwickelte Modell der 1-DollarBrille. Durch sein Konzept können auch die Ärmsten Zugang zu bezahlbaren Brillen bekommen. Aufmuth schreibt: Sie besteht aus einem leichten, flexiblen Federstahlrahmen und fertigen Gläsern aus Kunststoff, die einfach eingeklickt werden. Die 1-Dollar-Brille kann von den Menschen vor Ort selbst hergestellt und verkauft werden. Der Materialpreis liegt bei rund 1 US-Dollar, der Verkaufspreis bei 2-3 ortsüblichen Tageslöhnen. 


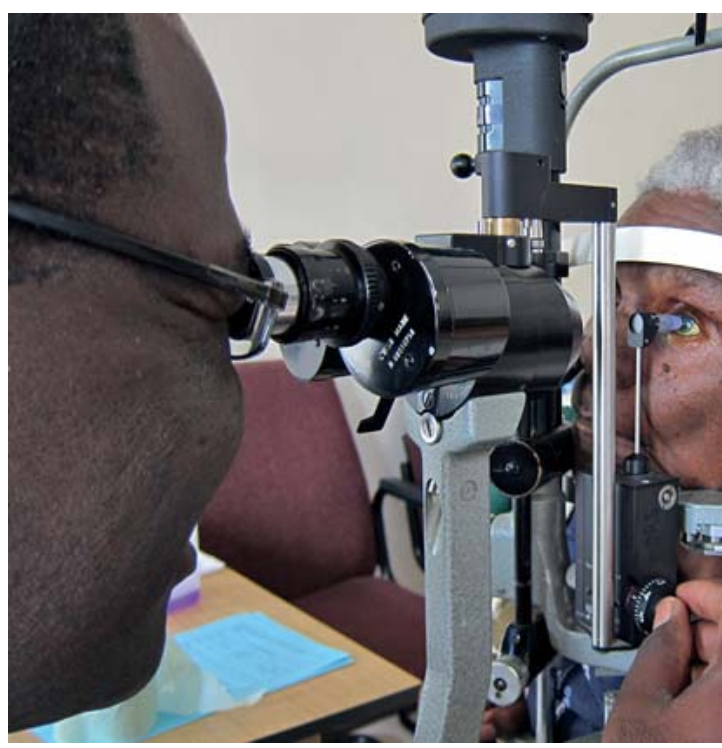

Abb. 7 Goldman-Applanations-Tonometrie bei einer Glaukompatientin in Malawi, Afrika.

\section{Der grüne Star - das Glaukom}

Das primäre Offenwinkelglaukom (POWG) ist der zweithäufigste Grund einer Erblindung in Afrika, der dritthäufigste Grund weltweit, verantwortlich für 10\% weltweiter Erblindung (Abb. 7). 2006 glaubte man annehmen zu können, dass 2010 60,5 Millionen Menschen vom Glaukom betroffen sein werden, wovon 8,4 Millionen erblindet sein würden. Quigley u. Broman wiesen 2006 darauf hin, dass 2010 wahrscheinlich 44,7 Millionen am POWG und 15,7 Millionen am primären Engwinkelglaukom (PEWG) erkrankt sein würden [11].

Merke: Wichtig in diesem Zusammenhang ist zu wissen, dass das PEWG zwar nur $26 \%$ aller Glaukomerkrankungen ausmacht, aber dennoch verantwortlich ist für fast die Hälfte aller an Glaukom erblindeten Menschen.

Das Glaukom ist der weltweit häufigste Grund einer irreversiblen Erblindung. Man schätzte 2006, dass bis zum Jahr 2020 ca. 80 Millionen Menschen am Glaukom erkrankt sein werden, d. h. eine Zunahme um 20 Millionen Betroffene seit 2010. Außerdem galt 2006, dass bis 20108 Millionen Menschen am Glaukom bilateral erblindet sein würden und deren Zahl bis 2020 mit steigender Prävalenz auf über 11 Millionen steigen würde, es sei denn, verbesserte Screeningmethoden und effektive Behandlungsstrategien würden ihre Wirkung zeigen. In SSA wird es jedoch schwierig bleiben, allen identifizierten Glaukompatienten dies zukommen zu lassen. Hohe Kosten und eine geringe Patientencompliance zur kontinuierlichen Behandlung chronischer Erkrankungen verhindern dies. Die Mehrheit der Weltbevölkerung - und die der Patienten mit Glaukom - lebt in Entwicklungsländern. Die meisten von ihnen sind sich ihrer Krankheit nicht bewusst, obschon eine schwerere Sehbehinderung vorliegt. Das Risiko, nach einem Zeitraum von 12-20 Jahren durch das POWG zu erblinden, liegt bei $14,5-27 \%$ für unilaterale und bei 7-9\% für eine bilaterale Erblindung.

Das Glaukom kann nicht isoliert behandelt werden. Dessen Entdeckung und Behandlung muss in bereits bestehende Augenheilkundeprojekte durch stets komplette Augenuntersuchungen integriert werden. Es gehört deshalb in den Lehrplan aller augenheilkundlichen Facharztausbildungen.

Merke: Wichtig ist, dass jeder Ophthalmic Technician, jeder OCO und jeder Augenarzt wenigstens „das Glaukom bei jedem Menschen erkennt, der ihm als Patient gegenübersitzt“.

Dies gilt auch für alle augenärztlichen Untersuchungen in Industrieländern, wo man schätzt, dass jedes zweite Glaukom trotz Vorstellung beim Augenarzt nicht erkannt wird.

Denkt man an die Pyramide (Abb. 3) und ihre erfolgreiche Implementierung in Andhra Pradesh, Indien, so ist ein wesentlicher Erfolgsfaktor zur Entdeckung von Frühzeichen glaukomatöser Augenveränderungen die Einführung der Frequenzverdoppelungsperimetrie (engl. Frequency Doubling Perimetry Technique, FDP) auf dem Niveau der Vision Center, die zum Sektor der Primärversorgung gehören.

Thomas u. Mitarb. publizierten, dass ein Vision Technician an einem Vision Center einen positiven Vorhersagewert okularer Pathologien von ca. 50\% mit der FDT erreichte und sie somit zum nächsthöheren Center weiterleitete [12]. Es konnte 2014 gezeigt werden, dass Kandidaten ohne Vorbildung in Bezug auf das Krankheitsbild Glaukom allein anhand der FDP-Ausdrucke die gleichen diagnostischen Ergebnisse erzielen konnten wie augenheilkundlich vorgebildete Kandidaten.

Schaut man sich den komplexen Prozess der Entdeckung, der Diagnosestellung und Behandlung des Glaukoms an, so kommt man zu dem Schluss, dass Glaukomprogramme nur initiiert werden sollten, wenn die diagnostischen und operativen Möglichkeiten vor 
Ort sind. In Phase 1 von VISION 2020 (1999-2010) hat man sehr viel Wert auf die Einführung von MSICS und gute chirurgische Ergebnisse gelegt. In der zweiten Hälfte von VISION 2020 (2010 - 2020), in der auch das Glaukom und die diabetische Retinopathie als Erblindungsursachen mit besonderem Fokus behandelt werden sollen, kommt es darauf an, ebenfalls die richtigen Rahmenbedingungen in geografisch definierten Gebieten zu schaffen.

Es ist ebenfalls notwendig, dass MSICS-Chirurgen eine Trabekulektomie (TET) operieren können. Sie sollten auch mit einer Lasertrabekuloplastik oder einer transskleralen Cyclophotokoagulation vertraut sein. Wichtig in Bezug auf das PEWG ist die Entfernung der klaren Linse mit oder ohne TET. Derzeit wird die Effektivität beider Verfahren eingehend untersucht.

\section{Diabetische Retinopathie}

Es gibt eine Epidemie sog. nichtübertragbarer Krankheiten (engl. non-communicable diseases, NCD), zu denen u.a. die Zuckererkrankung zählt. Sie ist die führende Ursache der Erblindung in der arbeitsfähigen Bevölkerung weltweit. Gleichzeitig gibt es eine hohe Zahl nichtdiagnostizierter Fälle an Diabetes mellitus. Die Prognose für 2030 ist, dass weltweit 5\% der Menschen an Diabetes leiden werden (522 Millionen), falls keine wirksamen Anstrengungen unternommen werden, was in ca. 5\% zu Erblindungen durch die proliferative diabetische Retinopathie führen wird. In Afrika wird sie erklärt als eine Folge der Verstädterung, des geänderten Lebensstils, einer Fehlernährung, einer alternden Bevölkerung und Umweltfaktoren. Nach Angaben des Diabetes-Weltverbands lebten 2012 in Afrika ungefähr 14,7 Millionen Menschen mit Diabetes und hochgerechnet wird sich deren Zahl - falls der Trend dazu so anhält - bis 2030 verdoppeln (28 Millionen).

Shaw u. Mitarb. haben berechnet, dass zwischen 2010 und 2030 die Anzahl der Diabetiker in Entwicklungsländern um 69\%, in Industrienationen um 20\% zunehmen wird, das bedeutet für Mittel- und Niedrigeinkommensländer ein Zuwachs von 291 Millionen Diabetikern im Jahr 2011 auf 458 Millionen im Jahr 2030 [13].

Die diabetische Retinopathie als eine Folge retinaler mikrovaskulärer Komplikationen wird in der milden Form als nichtproliferative diabetische Retinopathie

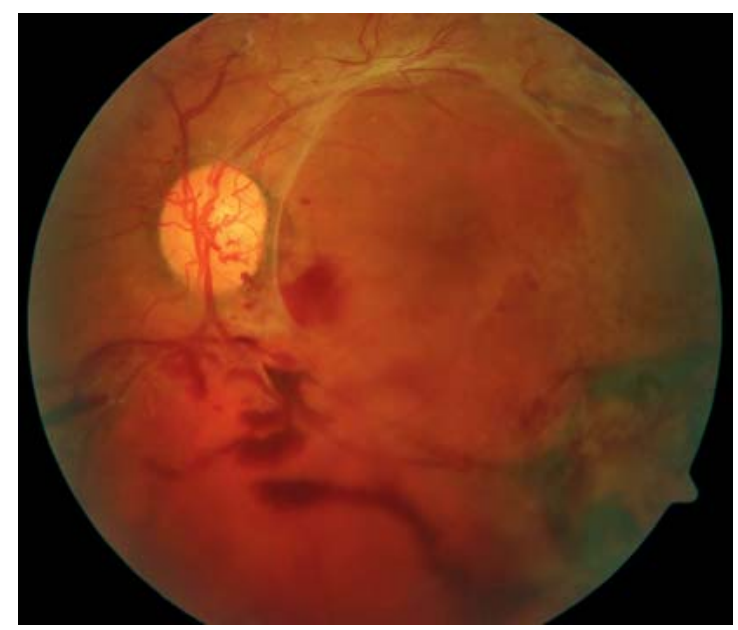

Abb. 8 Proliferative diabetische Retinopathie (PDR).

(NPDR), in der schwereren als proliferative diabetische Retinopathie (PDR) bezeichnet (Abb. 8). Die diabetische Makulopathie ist allerdings der häufigste Grund eines Sehverlusts bei Diabetikern. Somit ist sie auch eine oft auftretende Komplikation in Entwicklungsländern.

In einer Diabetes-Sprechstunde in Malawi hatten $50 \%$ der Diabetiker eine diabetischen Retino- bzw. Makulopathie, $30 \%$ von ihnen waren dadurch schon in ihrem Sehen eingeschränkt. Die Prävalenz einer diabetischen Retino- bzw. Makulopathie ist damit in Malawi fünfmal so hoch wie in europäischen Studien gezeigt werden konnte, das Risiko einer Sehbehinderung durch dieselbe ist dreimal so hoch. Diese hohe Prävalenz wird mit der unterdurchschnittlich schlechten Kontrolle des Blutglukosespiegels und des Blutdrucks begründet. Auch fehlt es an Programmen, eine diabetische Retinopathie zu diagnostizieren bzw. zu behandeln.

Screeninguntersuchungen, frühes Diagnostizieren und Behandeln der diabetischen Retinopathie sind die Eckpfeiler, um einen Sehverlust zu vermeiden. In einigen asiatischen (Nepal, Indien) und afrikanischen Ländern wie Botswana, Sambia, Tansania und Ghana sind dementsprechende Programme initiiert worden. Sie stecken jedoch noch in den Kinderschuhen und haben mit vielen Schwierigkeiten zu kämpfen, denn die Gesundheitssysteme in Afrika sind häufig als „On-off-Treatment“ (einmal kommen, einmal ambulant behandeln, nach Hause gehen) konzipiert. Um Katarakte zu operieren oder eine Brille anzupassen ist dies hilfreich, jedoch nicht bei der Behandlung chronischer Krankheiten. 
In vielen Ländern gibt es finanzielle Verteilungsprobleme hinsichtlich sog. übertragbarer Krankheiten wie Malaria und Tuberkulose im Wettstreit mit den NCDs. Es fehlt zudem an Unterstützungssystemen wie Computern mit entsprechender Software, um effiziente Strukturen zur Behandlung chronischer Krankheiten aufzubauen.

\section{Kinderblindheit (Childhood blindness, CHB)}

Als VISION 2020 im Jahr 1999 als weltweite Initiative ausgerufen wurde, ging man von 1,4 Millionen erblindeten Kindern aus. Die Annahme war, dass es bis 2020 2 Millionen werden könnten. 40\% der Erblindungen im Kindesalter galten als vermeidbar. Als Hauptursachen einer Erblindung galten:

- korneale Erblindung,

- kindliche Katarakt,

- Frühgeborenenretinopathie (ROP),

- unkorrigierte Refraktionsfehler.

Ursachen der kornealen Erblindung waren:

- Vitamin-A-Mangel (engl. Vitamin A Deficiency, VAD),

- Masern,

- Ophthalmia neonatorum,

- schädliche Infektionen durch traditionelle Medizin, die oft Pflanzensäfte verwendet,

- andere korneale Infektionen.

Die Verantwortlichen von WHO und IAPB entwickelten eine integrierte Strategie zur Verhütung der CHB auf allen drei Ebenen der Augenheilkunde (Tab. 5).

Da viele Erblindungsursachen eng mit der Sterblichkeit von Kindern korrelieren, war es offenkundig, dass, wenn die Unter-5-Jahre-Sterblichkeitsrate (Under-5Mortality Rates, U5MRs) gesenkt werden könnte, auch die Kindererblindung sinken würde. Ursachen dieser Sterblichkeit können u.a. Masern, Vitamin-A-Mangel und kongenitale Röteln sein, die ihrerseits auch für eine hohe Prävalenz der Kinderblindheit verantwortlich sind. 2010 konnten Chandna u. Gilbert aufzeigen, dass die Kinderblindheit weltweit um 10\% abgenommen hat [14]. Zum gleichen Zeitpunkt war sie aber in SSA um $30 \%$ gestiegen, denn auch die U5MR war wegen der HIV-Epidemie und opportunistischer Infektionen deutlich gestiegen, was zu mehr Erblindung führte.

\section{Tabelle 5}

Integrierte Strategie der drei Gesundheitssektoren, um Kinderblindheit zu verhüten.

\begin{tabular}{|c|c|}
\hline Ebene & Behandlungsstrategie \\
\hline tertiärer Sektor & $\begin{array}{l}\text { Chirurgie und Behandlung der kongenitalen Katarakt, ROP, } \\
\text { kornealen Trübung, Glaukom usw. } \\
\text { Ausbildung von Pflegern, HCW usw. } \\
\text { Bereitstellung von vergrößernden Sehhilfen } \\
\text { Forschung }\end{array}$ \\
\hline sekundärer Sektor & $\begin{array}{l}\text { Identifikation und Behandlung von Refraktionsfehlern, } \\
\text { Erkrankungen des äußeren Auges, Überweisung komplexerer } \\
\text { Pathologien }\end{array}$ \\
\hline primärer Sektor & $\begin{array}{l}\text { Prävention kornealer Vernarbung durch Masernimpfung; } \\
\text { Ernährungslehre zur Verhinderung VAD usw. } \\
\text { frühe Behandlung einfacher Augenprobleme } \\
\text { Prävention von Röteln durch Impfung } \\
\text { frühe Identifikation und Überweisung von Kindern mit } \\
\text { Krankheiten, die zu einer Sehbehinderung führen können }\end{array}$ \\
\hline
\end{tabular}

Um die Sterblichkeit durch Masern zu bekämpfen, initiierte die WHO 1974 ein ausgedehntes Impfprogramm (Expanded Programme of Immunization, EPI). Trotz dieser Maßnahme und einer Impfrate von $80 \%$ im Jahre 1992 starb immer noch 1 Million Kinder (2,4\%) pro Jahr von 41 Millionen an Masern erkrankten Kindern. Es ist so, dass bei Masern, die hoch infektiös sind, erst eine Durchimpfungsrate von $>90 \%$ zu einer schützenden Herdenimmunität führt. Zudem bietet die aktuell von der WHO empfohlene einmalige Masernimpfung im 1. Lebensjahr keinen anhaltenden Schutz bis ins Erwachsenenalter.

Fast 20 Jahre später untersuchte Sudfeld die Effektivität von Masernimpfung und gleichzeitiger Vitamin-AGabe. Vitamin-A-Mangel ist ein bekannter Risikofaktor für eine schwere Maserninfektion, weshalb die WHO seit 1987 die Masernimpfung mit einer gleichzeitigen Vitamin-A-Gabe empfahl. Sudfeld konnte erschließen, dass seine Ergebnisse die neue Strategie von WHO/ UNICEF rechtfertigen [15]. Das Ziel der GIVS (Global Immunization Vision and Strategy) ist es, durch eine zweifache Masernimpfung in Südostasien die Mortalität durch Masern um bis zu 90\% zu senken. 


\section{Die kindliche Katarakt}

Heutzutage ist der häufigste Grund einer Erblindung im Kindesalter in SSA die kindliche Katarakt, denn das Auftreten kornealer Erblindung sank merklich nach Einführung der zuvor genannten Programme.

Mittlerweile gibt es im tertiären Sektor auch Subspezialisierungen für Kinderaugenheilkunde, sodass zumindest ein Kinderophthalmologe einer Bevölkerung von 50 Millionen bis 2010 zur Verfügung stehen sollte, bis 2020 sollte es einer für 10 Millionen sein. Doch allein eine Operation des kindlichen grauen Stars ist nicht ausreichend. Es bedarf der Nachsorge, der wiederholten Refraktion und Brillenanpassung über Jahre. Um möglichst gute postoperative Ergebnisse zu erzielen, wäre eine genauere Formel zur Kalkulation der präoperativ zu wählenden intraokularen Linse wünschenswert, wie Medsinge u. Nischal betonen [16]. Bisher erfolgt die IOL-Wahl entsprechend dem Lebensalter des Patienten und der zu erwartenden Myopisierung. Die meisten postoperativen Komplikationen entstehen durch den Nachstar oder das Sekundärglaukom.

In einer eigenen Studie in Malawi konnten wir herausarbeiten, dass, obwohl die kindliche Kataraktchirurgie kostenfrei angeboten wird, dieser Service nur zu einem Drittel von den Eltern und ihren Kindern nach ausführlicher Erstuntersuchung von Ärzten, Eltern und Kind wahrgenommen wird [17]. Der harte Arbeitsalltag und der lange Weg zu einem großen Krankenhaus verringern die Akzeptanz des Angebotes, d.h. dass ein allein kostenloses Angebot immer noch nicht ausreichend ist, um Kinder und deren Eltern im ländlichen Afrika von der Notwendigkeit einer oder mehrerer Interventionen zu überzeugen.

Merke: Deshalb ist die Bekämpfung von Blindheit nicht allein ein augenärztliches Problem. Es geht darum, bereits entstandene und neu entstehende Programme zur Augengesundheit in die allgemeine Gesundheitsversorgung zu integrieren.

Clare Gilbert, Beraterin von Sight Savers International (SSI), erwähnte schon 2001 wesentliche Elemente des primären Gesundheitswesens (Primary Health Care, PHC) in Entwicklungsländern [18]:

- Gesundheitserziehung,

- gute Ernährung,

- Vorhaltung der wichtigsten Medikamente,

- frisches Wasser,

- gute Sanitäranlagen,
- Kontrolle endemischer Krankheiten,

- die Behandlung der einschlägigen Krankheiten.

Es ist ein kontinuierlicher Kampf, um geringere Inzidenzen zu erzielen. Die derzeitige Prävalenz der CHB liegt bei 0,4/1000 Lebendgeburten in Industrieländern und bis zu 1,5/1000 in armen Ländern. Bis heute werden Impfprogramme für Röteln und Masern benötigt, um die Inzidenz der CHB weltweit zu reduzieren.

\section{Frühgeborenenretinopathie (ROP)}

Nach ICD-Klassifikation (International Classification of Diseases) kann die Frühgeborenenretinopathie (ROP) eine potenziell das Sehen bedrohende Erkrankung sein, die zur Amblyopie, zu Strabismus, Refraktionsfehlern oder Sehbehinderung führt. Die ROP ist ein multifaktorielles Geschehen. Wichtige pathogenetische Faktoren sind das Gestationsalter bei Geburt sowie das Geburtsgewicht, postnatale Faktoren wie Sauerstoffexposition, Gewichtszunahme und die Expression angiogener Wachstumsfaktoren. Am Auge kann es zu visusbedrohenden retinalen Neovaskularisationen kommen, im ungünstigsten Fall zu einer Netzhautablösung. Die allermeisten Fälle sind jedoch mild und bilden sich von selbst zurück. Eine fortgeschrittene ROP kann jedoch zu einer Netzhautablösung oder gar zum völligen Visusverlust führen. Viele betroffene Kinder leiden gleichzeitig an neurologischen Defiziten.

Blencowe u. Kollegen haben einmal regional und weltweit die Sehbehinderung frühgeborener Kinder 2010 untersucht [19]. Die wissenschaftliche Basis dazu war ein systematisches weltweites Literaturreview: Sie fanden heraus, dass 201013 Millionen (87\%) von 15 Millionen Frühgeborenen überlebten. 1,4\% von ihnen hatten eine milde ROP, 0,4\% eine fortgeschrittene ROP, die als potenziell visusbedrohend eingestuft wurde. Die Autoren schätzen, dass 2010 ca. 30000 (0,2\%) Frühgeborene weltweit durch die ROP sehbehindert wurden. Präventionsmaßnahmen müssen deshalb gelehrt werden, besonders die postpartal richtig dosierte Sauerstoffgabe. Screening und Behandlung der ROP bedürfen einer guten Zusammenarbeit zwischen Ophthalmologen, Neonatologen und Pädiatern. 


\section{Hornhautentzündung}

In Entwicklungsländern galt die Hornhautentzündung lange Zeit als eine Epidemie, die zur Erblindung führt. Jährlich sind weltweit mindestens 1,5 Millionen Menschen davon betroffen. Die tatsächliche Inzidenz der Erkrankung wurde 1993 in Südindien als zehnmal höher eingeschätzt als die in den USA. In Malawi erforschte Prof. Chirambo, dass sie bis zu 55\% aller Fälle monokularer Erblindung verursacht. In den 1980erJahren des vergangenen Jahrhunderts war monokulare Blindheit in Malawi zweimal häufiger anzutreffen als die Erblindung durch den grauen Star.

Die mikrobielle Keratitis kann durch Bakterien, Pilze, Viren oder Protozoen verursacht werden. Die Keime sind regional in unterschiedlicher Häufigkeit anzutreffen. Viren sind als Auslöser einer Keratitis eher in gemäßigten Klimazonen anzutreffen, wohingegen Hornhautulzera in tropischen Ländern meist durch Bakterien oder Pilze hervorgerufen werden, speziell Aspergillus und Fusarium. Die oberflächliche Hornhautverletzung als Folge eines pflanzlich induzierten Mikrotraumas ist oft bei Kleinbauern anzutreffen. Sie ist für bis zu 50\% aller Ulzera in Entwicklungsländern verantwortlich.

Das Ziel der Therapie ist, die Erreger zu eliminieren und den Schaden für die Hornhaut zu begrenzen. Dies ist jedoch eine große Herausforderung.

Burton berichtet, dass $41 \%$ der Patienten zum Zeitpunkt der Erstvorstellung in einem tansanischen Krankenhaus bereits einen Durchmesser von $5 \mathrm{~mm}$ des Hornhautulkus vorwiesen [20]. An Zentren tertiärer Versorgung kommen Patienten zumeist auch mit deutlich fortgeschrittenem Befund. Die lange Anreise, eine zeitweilig falsche Behandlung mit oft pflanzlicher traditioneller Medizin oder eine falsche antiinflammatorische Medikation mit Steroiden haben dazu beigetragen.

\section{HIV und das Auge}

2013 waren weltweit 35 Millionen Menschen mit HIV infiziert. 70\% von ihnen leben in SSA. Die HIV-Infektion samt assoziierten Erkrankungen stellt die Gesundheitssysteme vor große Probleme, und auch die assoziierten Augenerkrankungen können nicht immer adäquat behandelt werden. Es ist ja neben den Patienten

\section{Tabelle 6}

HIV-assoziierte Erkrankungen in Relation zum Immunstatus (Daten aus [21]).

\begin{tabular}{|c|c|c|}
\hline HIV-Status & CD4-Zellen $/ \mathrm{mm}^{3}$ & assoziierte Erkrankung \\
\hline Serokonversion & 1000 & $\begin{array}{l}\text { Bindehautentzündung, Trockenes Auge, } \\
\text { retroorbitaler Schmerz }\end{array}$ \\
\hline frühe HIV-Infektion & $500-1000$ & $\begin{array}{l}\text { allergische Konjunktivitis, } \\
\text { Reiter-Syndrom, Uveitis intermedia, } \\
\text { retinale Vaskulitis, HIV-Retinopathie, } \\
\text { Neuritis nervi optici (NNO) }\end{array}$ \\
\hline $\begin{array}{l}\text { fortgeschrittene } \\
\text { HIV-Infektion }\end{array}$ & $200-500$ & $\begin{array}{l}\text { Trockenes Auge, Blepharitis, bakterielle } \\
\text { und folliluläre Konjunktivitis, Kaposi- } \\
\text { Sarkom, Molluscum contagiosum, } \\
\text { Herpes zoster, Herpes simplex, } \\
\text { HIV-Retinopathie, Uveitis granulo- } \\
\text { matosa (Tbc), Aspergillose }\end{array}$ \\
\hline späte HIV-Infektion & $0-200$ & $\begin{array}{l}\text { opportunistische Infektionen und } \\
\text { Tumoren, die alle okulären Strukturen } \\
\text { betreffen können }\end{array}$ \\
\hline
\end{tabular}

oft auch das Gesundheitspersonal betroffen. Beide Gruppen sterben an der Erkrankung und dadurch verschlimmert sich der Personalmangel im Gesundheitswesen noch einmal.

Sobald die Immunitätslage schwächer wird, können opportunistische Augenkrankheiten auftreten.

Tab. 6 zeigt HIV-assoziierte Erkrankungen in Relation zum Immunstatus. In diesem Artikel werden wir uns auf die Zytomegalovirus-Retinitis (CMV) und das Bindehautkarzinom (Ocular Surface squamous Neoplasia, OSSN) als okuläre Erkrankungen konzentrieren.

\section{Zytomegalovirus-Retinitis}

Das Zytomegalovirus ist ein Herpesvirus. Die primäre Infektion oder Reaktivierung einer latenten CMVInfektion im Rahmen einer Immunschwächekrankheit wie z.B. Aids führt zu Krankheiten an verschiedenen Organen:

- Gehirn (Meningoenzephalitis),

- Lunge (interstitielle Pneumonie),

- Gastrointestinaltrakt (Ösophagitis),

- Haut,

- periphere Nerven,

- Augen (CMV-Retinitis). 


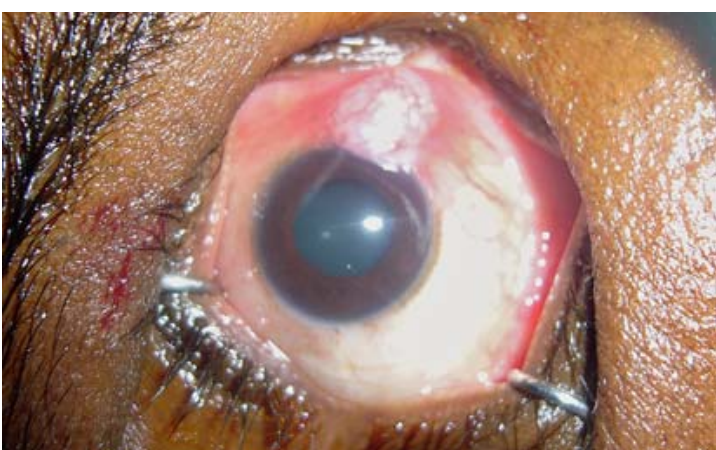

Abb. 9 OSSN - CIN.

Die CMV-Retinitis führt zur progressiven retinalen Nekrose. Die Retina erscheint dunkler, die entzündeten Gefäße zeigen Einscheidungen, es kommt zu intraretinalen Blutungen und einer milden Vitritis. Unbehandelt resultiert Blindheit. Die CMV-Retinitis war in industrialisierten Ländern die häufigste opportunistische Infektion, die zur Blindheit führte (25-40\% aller Aids-Patienten). Dies war in der Zeit, bevor die antiretrovirale Therapie (ART) zur Verfügung stand. In Entwicklungsländern war die CMV-Retinitis-Prävalenz regional sehr unterschiedlich.

In einer Multicenterstudie von HIV-Patienten aus Südafrika und Indien waren vitreoretinale opportunistische Infektionen (zumeist CMV) in Südafrika seltener als in Indien (2,6\% vs. 11,4\%) [22]. Die Autoren der Studie meinten dafür genetische Faktoren als eine mögliche Erklärung verantwortlich machen zu können.

Die Behandlung der CMV-Retinitis besteht aus einer Aufdosierungs- und einer Aufrechterhaltungsphase. Letztere wird so lange durchgeführt, bis sich das Immunsystem durch die ART erholt hat. Bevorzugte Medikamente sind Ganciclovir (i.v. oder intravitreal) oder Valganciclovir als orale Gabe. Die vitreoretinale Chirurgie mag indiziert sein, sobald die CMV-Retinitis zu einer Netzhautablösung führt.

In einigen Entwicklungsländern, wie z. B. Myanmar, wo die CMV-Retinitis unter Aids-Patienten besonders hoch ist, hat man Internisten dazu ausgebildet, die Erkrankung mittels direkter Ophthalmoskopie bzw. anschließender intravitrealer Injektionen zu diagnostizieren und zu behandeln. Die Kollegen machen regelmäßig Funduskopien bei allen fortgeschrittenen Fällen einer Immunsuppression (CD4-Zellen $<100$ Zellen $/ \mathrm{mm}^{3}$ ). Diese Intervention hat sich als besonders effektiv hinsichtlich der Diagnosestellung und auch der Behandlung gezeigt.

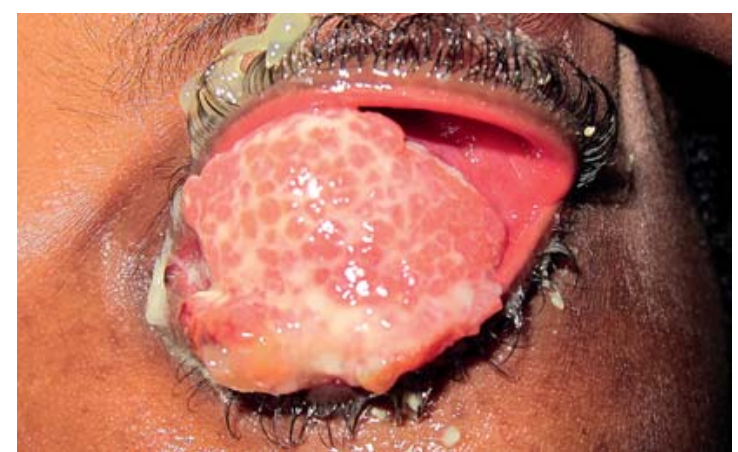

Abb. 10 OSSN - Orbita mit befallen.

\section{Das Bindehautkarzinom}

Die Bindehautdysplasie (Ocular Surface squamous Neoplasia, OSSN) gehört zum Spektrum präkanzeröser und kanzeröser Läsionen der Bindehaut. Dies reicht von konjunktivaler intraepithelialer Neoplasie (CIN) bis zu einem invasiven Tumor, der durch die Orbita bis in den Schädel dringen kann.

In Industrieländern ist es selten, in Entwicklungsländern und speziell im SSA häufig bei jungen Patienten anzutreffen. Die Prävalenz des OSSN wird auf 35 Personen/Millionen Bevölkerung pro Jahr im SSA geschätzt. Das mittlere Alter von Patienten mit OSSN in Simbabwe lag bei 35 Jahren.

OSSN-Risikofaktoren sind HIV-Infektion, Exposition gegenüber ultravioletter Strahlung und das Humane Papillomavirus (HPV).

In einer Studie in Malawi waren 79\% der OSSN-Patienten HIV-positiv, und die meisten dieser Patienten waren sich dessen nicht bewusst [23]. Mittlerweile gehört es in Malawi zur klinischen Routine, jedem OSSN-Patienten einen HIV-Test und eine anschließende Beratung bezüglich einer antiretroviralen Therapie anzubieten.

Die Patienten beklagen zunächst nur ein Fremdkörpergefühl, das Auge ist rot und man sieht ein wenig der CIN auf der Bindehaut (Abb. 9). Einzige Therapie, neben der systemischen ART, ist die chirurgische Exzision mit oder ohne Antimetaboliten (MMC oder 5-FU). Die größte Herausforderung besteht darin, dass die Patienten meist erst im weit fortgeschrittenen Stadium ärztliche Hilfe suchen. Wenn die Orbita wesentlich befallen ist (Abb. 10), bleibt oft nur die Enukleation des Bulbus, zusammen mit einer Exenteration des orbitalen Gewebes. Laut einer Studie von Nutt in Angola soll eine 
topische Behandlung mit 5-FU eine Alternative zur chirurgischen Entfernung einiger Fälle von CIN bei OSSN sein [24].

\section{Ausblick}

In diesem Beitrag haben wir Sie sowohl mit der Initiative VISION 2020 und dem Gobal Action Plan als auch mit speziellen Erkrankungen des Auges vertraut gemacht. Die Elimination vermeidbarer Blindheit ist möglich, allerdings braucht es dazu weitere länderspezifische Programme, die möglichst gut und schnell umgesetzt werden, um den Lichtlosen, wie Pastor Ernst Jakob Christoffel, der Gründer der Christoffel Blindenmission, die Blinden nannte, helfen zu können. VISION 2020 ist eine Initiative und kein starr vorgegebenes Programm, d.h.es kommt auf die Initiative jedes Einzelnen an - vielleicht auch auf Ihre?

Dein Auge gibt dem Körper Licht (Lukas 11, 34). Viele Augenärzte und alle in der Augenmedizin Tätigen sind von diesem Gedanken fasziniert und arbeiten mit Freude daran, immer wieder ein bisschen mehr Licht schenken zu können.

Interessenkonflikt: Dr. Schulze Schwering war im Rahmen der Universitätspartnerschaft zwischen der Universitätsaugenklinik Tübingen und dem Department of Ophthalmology der Universität Malawi als Lecturer zur Aus- und Weiterbildung von Fachärzten für eine Dauer von zwei Jahren entsendet (2011 - 2013). Seine Arbeit und die gleichzeitige apparative Modernisierung des Lions Sight First Eye Hospital (LSFEH), dem Lehrhospital der Universität Malawi, wurden von der Else KrönerFresenius-Stiftung getragen. Bis heute unterstützen der DAAD und die Else Kröner-Fresenius-Stiftung die Universitätspartnerschaft unter der Leitung des Teams um Professor Martin Spitzer, Universitätsaugenklinik Tübingen.

\section{Dieser Artikel erschien in den \\ Klinischen Monatsblättern für Augenheilkunde (DOI 10.1055/s-0033-1358176).}

\section{Quellenangaben}

1 Klauss V, Schaller UC. Tropenophthalmologie-Prävention und Therapie. Ophthalmologe 2004; 101: 741 - 763

2 Klauss V, Schaller UC. Internationale Initiativen zur Prävention von Blindheit. Ophthalmologe 2007; 104: 855-859

3 Nentwich MM, Schaller UC, Klauss V. Update: Diagnostik und Therapie von Tropenerkrankungen - Teil 1. Klin Monatsbl Augenheilkd 2009; 226: R139-151

4 Nentwich MM, Schaller UC, Klauss V. Update: Diagnostik und Therapie von Tropenerkrankungen - Teil 2. Klin Monatsbl Augenheilkd 2009; 226: R155-169

5 Investing in vision: Comparing the costs and benefits of eliminating avoidable blindness and visual impairment. Final report 2013. Im Internet: http://www.hollows.org; Stand: Juni 2015

6 Palmer JJ, Chinanayi F, Gilbert A et al. Trends and implications for achieving VISION 2020 human resources for eye health targets in 16 countries of sub-Saharan Africa by the year 2020. Hum Resour Health 2014; 12: 45

7 Kasper J, Bajunirwe F. Brain drain in sub-Saharan Africa: contributing factors, potential remedies and the role of academic medical centres. Arch Dis Child 2012; 97: 973 - 979

8 Foster A. Cataract and "Vision 2020 - the right to sight" initiative. Br J Ophthalmol 2001; 85: 635-637

9 Smith TS, Frick KD, Holden BA. Potential lost productivity resulting from the global burden of uncorrected refractive error. Bull World Health Organ 2009; 87: 431-437

10 Naidoo KS, Jaggernath J. Uncorrected refractive errors. Indian J Ophthalmol 2012; 60: 432-437

11 Quigley HA, Broman AT. The number of people with glaucoma worldwide in 2010 and 2020. Br J Ophthalmol 2006; 90: 262 -267

12 Thomas R, Naveen S, Nirmalan PK et al. Detection of ocular disease by a vision center technician \& the role of frequency doubling technology perimetry in this setting. $\mathrm{Br}$ J Ophthalmol 2010; 94: 214-218

13 Shaw JE, Sicree RA, Zimmet PZ. Global estimates of the prevalence of diabetes for 2010 and 2030. Diabetes Res Clin Pract 2010; 87: 4-14

14 Chandna A, Gilbert C. When your eye patient is a child. Community Eye Health 2010; 23: 1-3

15 Sudfeld CR1, Navar AM, Halsey NA. Effectiveness of measles vaccination and vitamin A treatment. Int J Epidemiol 2010; 39 (Suppl. 1): i48-i55

16 Medsinge A, Nischal KK. Pediatric cataract: challenges and future directions. Clin Ophthalmol 2015; 9: 77-90

17 Schulze Schwering M, Finger RP, Barrows J et al. Barriers to uptake of free pediatric cataract surgery in Malawi. Ophthalmic Epidemiol 2014; 21: $138-143$

18 Gilbert C, Foster A. Childhood blindness in the context of VISION 2020 - the right to sight. Bull World Health Organ 2001; 79: $227-232$

19 Blencowe H, Lawn JE, Vazquez T et al. Preterm-associated visual impairment and estimates of retinopathy of prematurity at regional and global levels for 2010. Pediatr Res 2013; 74 (Suppl. 1): $35-49$

20 Burton MJ, Pithuwa J, Okello E et al. Microbial keratitis in East Africa: why are the outcomes so poor? Ophthalmic Epidemiol 2011; 18: $158-163$ 
21 Mbcluskey PJ. Overview of HIV infection and pre-AIDS ocular manifestations. In: Lightman S, ed. HIV and the Eye. London: Imperial College Press; 2000

22 Pathai S, Gilbert C, Weiss HA et al. Differing spectrum of HIV-associated ophthalmic disease among patients starting antiretroviral therapy in India and South Africa. Trop Med Int Health 2011; 16: $356-359$

23 Spitzer MS, Batumba NH, Chirambo T et al. Ocular surface squamous neoplasia as the first apparent manifestation of HIV infection in Malawi. Clin Experiment Ophthalmol 2008; 36: 422-425

24 Nutt RJ, Clements JL, Dean WH. Ocular surface squamous neoplasia in HIV-positive and HIV-negative patients and response to 5-fluorouracil in Angola. Clin Ophthalmol 2014; 8: 2435-2440

\section{Zum Weiterlesen und Vertiefen}

25 Schulze Schwering M. Global blindness. Ophthalmologe 2007; 104: $845-848$

26 Resnikoff S, Pascolini D, Mariottia SP et al. Global magnitude of visual impairment caused by uncorrected refractive errors in 2004. Bull World Health Organ 2008; 86: 63-70

27 Ackland P. The accomplishments of the global initiative VISION 2020: The Right to Sight and the focus for the next 8 years of the campaign. Indian J Ophthalmol 2012; 60: 380-386

28 Schulze Schwering M, Spitzer MS, Kalua K et al. Training ophthalmologists for developing economies: an African-German partnership. Postgrad Med J 2014; 90: 61 -62

29 IAPB. Global action plan 2014. Im Internet: http://www.iapb.org/ advocacy/who-action-plan; Stand: Juni 2015

30 Rao GN. The Barrie Jones Lecture-Eye care for the neglected population: challenges and solutions. Eye (Lond) 2015; 29: 30-45

\section{Weitere Literatur beim Verfasser.}

\section{Korrespondenzadresse}

Dr. med. Markus Schulze Schwering, FEBO

Universitätsaugenklinik Tübingen

Schleichstraße 12-16

72076 Tübingen

Telefon: 07071/29-83721

Fax: 07071/29-3730

E-Mail: mssoculus@web.de 


\section{CME-Fragen}

\section{CME.thieme.de}

CME-Teilnahme

- Viel Erfolg bei Ihrer CME-Teilnahme unter http://cme.thieme.de

- Bitte informieren Sie sich über die genaue Gültigkeitsdauer unter http://cme.thieme.de

- Sollten Sie Fragen zur Online-Teilnahme haben, unter http://cme.thieme.de/hilfe finden Sie eine ausführliche Anleitung.

\section{1}

Auf welchen Zielen beruht die Initiative VISION 2020?
A Kontrolle wesentlicher Augenerkrankungen, Aufbau einer Infrastruktur, Aus- und Weiterbildung von Augenärzten und Hilfspersonal.

B Gründung von WHO-Augenkliniken.

C Integration von lokaler Augenheilkunde in Landesgesundheitsprogramme.

D Errichtung von Komitees zur Verhütung von Blindheit.

E Errichtung von mindestens 1 Blindenschule pro 1 Million Einwohner.

\section{2}

Was ist das übergeordnete Ziel des Globalen Aktionsplans von WHO und IAPB für die Jahre 2014-2019?
A Weisungsbefugnis der WHO über alle Gesundheitsministerien der Entwicklungsländer.

B $4 / 5$ aller Erblindungen zu vermeiden.

C Zugang zur Augenheilkunde für jeden.

D 25\% Verringerung von vermeidbarer Blindheit und Sehbehinderung bis 2019 auf der Basis der Daten von 2010.

E Freie Heilfürsorge in allen Augenkliniken der Entwicklungsländer.
3

Welche Aussage ist richtig? Das „Pyramidal Eye Care Service Delivery Model“....
A wurde von WHO und IAPB entwickelt.

B ist in einem indischen Bundesstaat erfolgreich implementiert worden, um auch den ärmsten Patienten im entlegensten Hinterland mit qualitativ hochwertiger Augenheilkunde zu versorgen.

C soll weltweit in gleicher Weise von den Gesundheitsbehörden kopiert werden.

D ist eine $\mathrm{GmbH}$ von Augenärzten und Optikern, um hohe Renditen zu erzielen.

E soll ein börsennotiertes Unternehmen werden.

4

Welche Aussage ist richtig? Der Zugewinn an Produktivität, der durch gutes Sehen in der arbeitenden Bevölkerung (15-65 Jahre) weltweit entstünde, ...
A liegt um $50 \%$ unter den Investitionskosten und ist deshalb zu teuer.

B liegt zweimal über den Investitionskosten, und nur ein Drittel der weltweiten Gesamtkosten werden von den Entwicklungsländern benötigt.

C liegt dreimal über den Investitionskosten, und zwei Drittel der weltweiten Gesamtkosten werden von den Industrieländern benötigt.

D ist so gering, dass Augengesundheit im Vergleich zur Aids-Bekämpfung von der WHO herabgestuft wurde.

E liegt bei fast 400 Milliarden US-Dollar.

5

Welche Antwort ist richtig? Welche sind die Mindestvorgaben an Personal für Augenheilkunde pro 1 Million Bevölkerung für das subsaharische Afrika?
A 1 Augenarzt, 5 Kataraktchirurgen, Techniker und Pfleger, 10 Optometristen und Refraktionisten.

B 10 Augenärzte, 50 Kataraktchirurgen, Techniker und Pfleger, 100 Optometristen und Refraktionisten.

C 4 Augenärzte, 10 Kataraktchirurgen, Techniker und Pfleger, 20 Optometristen und Refraktionisten.

D 8 Augenärzte, 20 Kataraktchirurgen, Techniker und Pfleger, 40 Optometristen und Refraktionisten.

E 16 Augenärzte, 40 Kataraktchirurgen, Techniker und Pfleger, 60 Optometristen und Refraktionisten. 


\section{CME-Fragen}

\section{6}

Welche Aussage ist richtig? Die Ausbildung von Augenärzten in ihren afrikanischen Heimatländern (11 von 12 Monaten), gepaart mit vierwöchigen (1 von 12 Monaten) Aufenthalten an deutschen Universitätsaugenkliniken ...
A hat zu einem massiven Verbleib afrikanischer Augenärzte im deutschen Gesundheitssystem geführt.

B ist ein Modell mit „Low Brain Drain“, d. h. die afrikanischen Kollegen arbeiten zu einem hohen Prozentsatz in ihren Heimatländern.

C wird zum Großteil durch die Gesundheitsministerien afrikanischer Länder finanziert.

D ist preiswert und die Kosten werden von den Assistenzärzten selbst getragen.

E ist ein Prioritätsprogramm der deutschen Bundesregierung.

\section{7}

Welche Aussage ist richtig?

Der altersbedingte graue Star ...

A ist weltweit als Erblindungsursache Nummer Eins eliminiert.

B wird am besten als Erblindungsursache bekämpft bei hoher CSR (Cataract surgical Rate) und gleichzeitig hoher CSC (Cataract surgical Coverage).

C wird mittlerweile in Entwicklungsländern primär mit der Phakoemulsifikations-Methode operiert.

D ist in der Behandlung sehr teuer, da die MSICS (Manual small Incision Cataract Surgery) aufwendige, komplizierte Technik erfordert.

E wird in Entwicklungsländern zumeist ohne Implantation einer Hinterkammerlinse durchgeführt.

8

Welche Aussage ist richtig? Unkorrigierte Refraktionsfehler ...
A sind weltweit gesehen mit über $50 \%$ Hauptursache einer Sehbehinderung.

B können in Entwicklungsländern allesamt mit der sog. 1-Dollar-Brille behoben werden.

C sind unter Erwachsenen deutlich höher als bei Kindern.

D verursachen keinen nennenswerten Produktivitätsverlust.

E benötigen zur Behebung statt gut geschultem Personal nur die Bereitstellung von Autorefraktoren.

9

Welche Aussage ist richtig?
A Der grüne Star ist der weltweit häufigste Grund einer irreversiblen Erblindung.

B Weltweit haben $3 / 4$ aller an Glaukom erkrankten Menschen ein primäres Offenwinkelglaukom (POWG), 1/4 ein primäres Engwinkelglaukom (PEWG). Allerdings sind fast $50 \%$ aller Erblindungen am Glaukom auf das PEWG zurückzuführen.

C 3,15 Millionen Menschen waren 2010 am Glaukom erblindet.

D Bis zum Jahr 2020 werden ca. 80 Millionen Menschen weltweit am Glaukom erkrankt sein.

E Die Aussagen A-D sind richtig.

10

Welche Aussage zur

Kinderblindheit ist richtig?
A Im Jahre 1999 ging man von 1,4 Millionen blinden Kindern aus.

B $\quad 40 \%$ der Erblindungen bei Kindern galten 1999 als vermeidbar.

C Die korneale Erblindung von Kindern konnte durch Einführung der Masernimpfung und gleichzeitiger Vitamin-A-Gabe deutlich gesenkt warden.

D Heutzutage ist der häufigste Grund einer Erblindung im Kindesalter in SSA die kindliche Katarakt.

E Die Antworten A-D sind richtig. 